\title{
Unfractionated Heparin during the Interruption of Antiplatelet Therapy for Non-cardiac Surgery after Drug-eluting Stent Implantation
}

\author{
Akihito Tanaka ${ }^{1}$, Hideki Ishii ${ }^{1}$, Yosuke Tatami ${ }^{1}$, Yohei Shibata ${ }^{1}$, Naohiro Osugi ${ }^{1}$, \\ Tomoyuki Ota ${ }^{1}$, Yoshihiro Kawamura ${ }^{1}$, Susumu Suzuki ${ }^{1}$, Yoshimasa Nagao ${ }^{2}$, \\ Tadashi Matsushita $^{3}$ and Toyoaki Murohara ${ }^{1}$
}

\begin{abstract}
Objective Heparin is not recommended to be administered during the interruption of antiplatelet therapy for non-cardiac surgery. However, there are insufficient data to determine the value. The purpose of the present study was to evaluate the clinical results of the administration of unfractionated heparin during the interruption of antiplatelet therapy in non-cardiac surgery patients who had previously undergone drug-eluting stent (DES) implantation.

Methods We retrospectively identified 210 elective non-cardiac surgical procedures that were performed with the administration of unfractionated heparin during interruption of all antiplatelet therapies in patients who had previously undergone DES implantation. Heparin was administered during the perioperative period in accordance with the local practice guideline at out institution. We examined the clinical outcomes within 30 days of surgery.

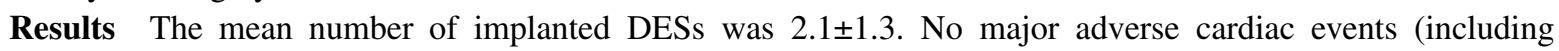
cardiac death, definite stent thrombosis, and non-fatal myocardial infarction) occurred in any of the 210 cases within 30 days of surgery. Four of the 210 cases $(1.9 \%)$ required reoperation for bleeding within 30 days of surgery.

Conclusion Our data showed the potential for the perioperative management with unfractionated heparin administration in Japanese patients who had previously undergone DES implantation who required noncardiac surgery with the interruption of all antiplatelet therapies.
\end{abstract}

Key words: unfractionated heparin, drug-eluting stent, antiplatelet therapy, non-cardiac surgery

(Intern Med 55: 333-337, 2016)

(DOI: 10.2169/internalmedicine.55.5495)

\section{Introduction}

The risk of adverse cardiac events is significantly increased in the week following non-cardiac surgery in comparison to any other week after drug-eluting stent (DES) implantation (1). The interruption of antiplatelet therapy (APT) for surgery is considered to be one of the main reasons for the increased risk (2-5). The optimal perioperative manage- ment of APT in patients undergoing non-cardiac surgery after DES implantation has not been clearly elucidated. Recent guidelines do not recommend the administration of heparin, which had been widely used in clinical practice as a "bridging therapy" for APT. However, the data on this issue are scarce and are not sufficient to determine the value (2, 6-9).

The purpose of this study was to evaluate the clinical results of heparin administration during the interruption of all APTs for the performance non-cardiac surgery in Japanese

${ }^{1}$ Department of Cardiology, Nagoya University Graduate School of Medicine, Japan, ${ }^{2}$ Department of Quality and Patients Safety, Nagoya University Hospital, Japan and ${ }^{3}$ Department of Transfusion Medicine, Nagoya University Hospital, Japan

Received for publication March 31, 2015; Accepted for publication May 17, 2015

Correspondence to Dr. Akihito Tanaka, akihito17491194@gmail.com 


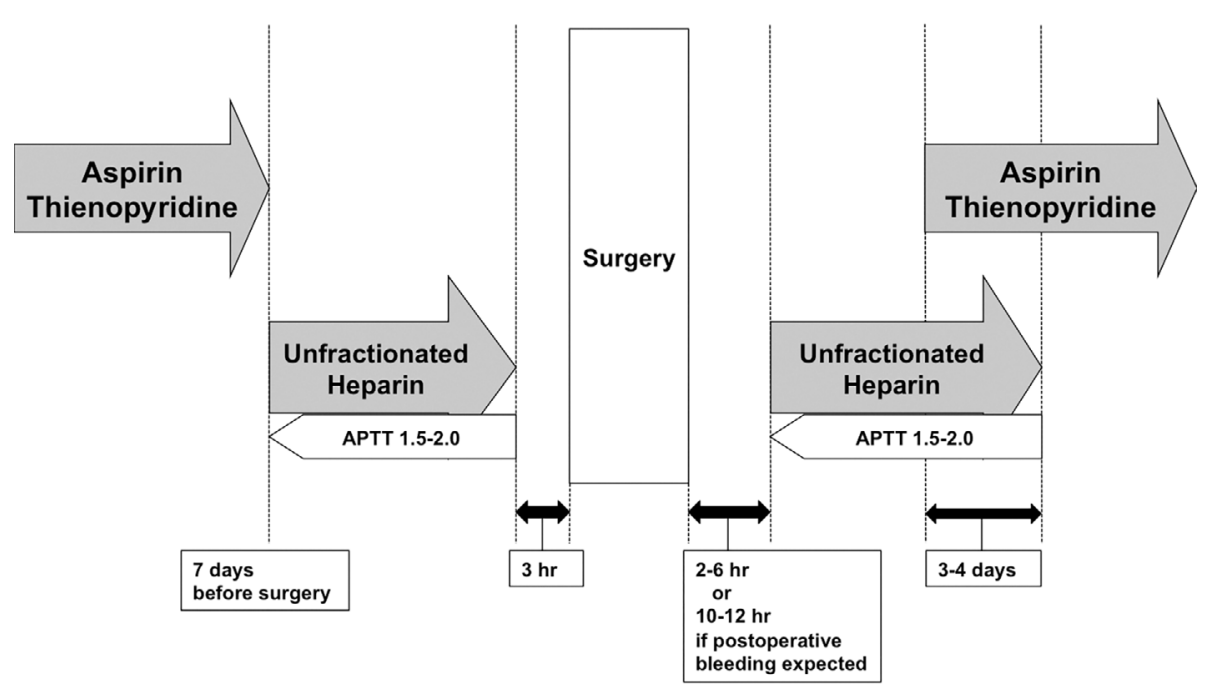

Figure. Heparin administration according to the local institutional practice guideline. Heparin administration was recommended for all DES patients who underwent non-cardiac surgery with the interruption of all APT agents regardless of the period after DES implantation. Continuous heparin administration was started at a rate of $200 \mathrm{U} / \mathrm{kg} / \mathrm{day}$ soon after the cessation of antiplatelet drugs. The dose was adjusted to maintain a patient-to-control ratio of activated partial thromboplastin time from 1.5 to 2.0, and the administration of heparin was stopped 3 hours before surgery. After surgery, heparin infusion was restarted as soon as possible (2-6 hours after the confirmation of hemostasis or 10-12 hours after the confirmation of hemostasis if postoperative bleeding was expected). The administration of heparin was continued until 3-4 days after APT was restarted.

patients who had previously undergone DES implantation.

\section{Materials and Methods}

We retrospectively identified 210 elective non-cardiac surgical procedures after DES implantation that required the interruption of all APT and were performed with the administration of heparin in the perioperative period at Nagoya University Hospital between January 2008 and October 2013. We excluded the patients who underwent surgery to treat active bleeding and emergency cases. Each attending surgical team administered heparin in accordance with the local institutional guideline regarding the provision of antithrombotic therapy. The local institutional guideline was as follows: the administration of heparin was recommended for all DES patients who underwent non-cardiac surgery with the interruption of all APT agents regardless of the length of time after DES implantation. Continuous heparin administration was started at a rate of $200 \mathrm{U} / \mathrm{kg} /$ day soon after the interruption of antiplatelet drugs. The dose was adjusted to maintain a patient-to-control ratio of activated partial thromboplastin time from 1.5 to 2.0 , and the administration was stopped 3 hours before surgery. After surgery, heparin infusion was restarted as soon as possible (2-6 hours after the confirmation of hemostasis, or 10-12 hours after the confirmation of hemostasis if postoperative bleeding was expected) and was continued until 3-4 days after APT was restarted (Figure). The practice guidelines did not refer to the administration of glycoprotein IIb/IIIa inhibitors because they had not been approved in Japan.

This study was performed according to the guidelines of the Declaration of Helsinki and was approved by the local Ethics Committee, which waived the requirement for written informed consent.

The first-generation DESs included sirolimus- and paclitaxel-eluting stents. The second-generation DESs included zotarolimus-, everolimus-, and biolimus-eluting stents. The types of surgical procedures that were performed were defined in accordance with the American College of Cardiology/American Heart Association guideline (8). Surgical hemorrhagic risk was categorized into 3 groups [low risk (transfusion normally not required); intermediate risk (transfusion frequently required); high risk (possible bleeding in a closed space)] (10).

The occurrence of major adverse cardiac events (MACE), stroke, and bleeding events within 30 days of the surgical procedures was investigated. MACE was defined as cardiac death, definite stent thrombosis, or non-fatal myocardial infarction. Stroke was defined as the newly-developed persistent loss of neurological function and an acute lesion identified with magnetic resonance imaging. For bleeding events, the requirement of postoperative transfusion and reoperations for bleeding were recorded. Bleeding events were also evaluated on the basis of thrombolysis in myocardial infarction (TIMI) bleeding classification [major bleeding: intracranial hemorrhage ( $>5 \mathrm{~g} / \mathrm{dL}$ decrease in hemoglobin concentration, or a $>15 \%$ absolute decrease in the hematocrit count); minor bleeding: observed blood loss (>3 g/dL de- 
Table 1. Baseline Characteristics at Surgical Procedure.

\begin{tabular}{|c|c|}
\hline & $\mathrm{n}=210$ \\
\hline \multicolumn{2}{|l|}{ Characteristics at surgical procedure } \\
\hline Age, y & $71 \pm 8$ \\
\hline Male, $n(\%)$ & $177(84.3 \%)$ \\
\hline Hypertension, $n(\%)$ & $159(75.7 \%)$ \\
\hline Dyslipidmia, n(\%) & $181(86.2 \%)$ \\
\hline Diabetes, $\mathrm{n}(\%)$ & $73(34.8 \%)$ \\
\hline Current smoker, n(\%) & $17(8.1 \%)$ \\
\hline $\mathrm{eGFR}<60 \mathrm{~mL} / \mathrm{min} / 1.73 \mathrm{~m}^{2}, \mathrm{n}(\%)$ & $93(44.3 \%)$ \\
\hline Hemodialysis, $\mathrm{n}(\%)$ & $6(2.9 \%)$ \\
\hline Prior myocardial infarction, $\mathrm{n}(\%)$ & $74(35.2 \%)$ \\
\hline Prior $\mathrm{CABG}, \mathrm{n}(\%)$ & $10(4.8 \%)$ \\
\hline No of vessel implanted DES (1/2/3/unknown), n & $131 / 65 / 9 / 5$ \\
\hline \multicolumn{2}{|l|}{ Treated coronary artery } \\
\hline Right coronary artery & 78 \\
\hline Left anterior descending artery & 120 \\
\hline Left circumflex artery & 71 \\
\hline Left main trunk & 21 \\
\hline Unknown & 6 \\
\hline No of DES implanted before, $n$ & $2.1 \pm 1.3$ \\
\hline \multicolumn{2}{|l|}{ Type of DES, $\mathrm{n}$} \\
\hline 1st-generation DES & 147 \\
\hline 2nd-generation DES & 35 \\
\hline Both & 12 \\
\hline Unknown & 16 \\
\hline Ejection fraction, $\%$ & $63 \pm 10$ \\
\hline \multicolumn{2}{|l|}{ Medications before surgical procedure } \\
\hline Aspirin, n(\%) & $189(90.0 \%)$ \\
\hline Thienopyridine, $\mathrm{n}(\%)$ & $148(70.5 \%)$ \\
\hline Aspirin + Thienopyridine, $\mathrm{n}(\%)$ & $129(61.4 \%)$ \\
\hline Cilostazol, n(\%) & $23(11.0 \%)$ \\
\hline Warfarin, $\mathrm{n}(\%)$ & $20(9.5 \%)$ \\
\hline Direct inhibitor of factor Xa, $\mathrm{n}(\%)$ & $1(0.5 \%)$ \\
\hline Statin, $\mathrm{n}(\%)$ & $163(77.6 \%)$ \\
\hline$\beta$-brocker, $\mathrm{n}(\%)$ & $94(44.8 \%)$ \\
\hline ACEI/ARB, n(\%) & $144(68.6 \%)$ \\
\hline Ca-antagonist, $\mathrm{n}(\%)$ & $105(50.0 \%)$ \\
\hline Nicorandil, $\mathrm{n}(\%)$ & $37(17.6 \%)$ \\
\hline Nitrate, $n(\%)$ & $30(14.3 \%)$ \\
\hline \multicolumn{2}{|c|}{$\begin{array}{l}\text { eGFR: estimated glomerular filtration rate, CABG: coronary } \\
\text { artery bypass grafting, DES: drug eluting stent, ACEI: } \\
\text { angiotensin-converting enzyme inhibitor, ARB: angiotensin } \\
\text { receptor II brocker }\end{array}$} \\
\hline
\end{tabular}

Table 3. Clinical Events within 30 Days of Surgery.

\begin{tabular}{ll}
\hline & $\mathrm{n}=210$ \\
\hline MACE, $\mathrm{n}(\%)$ & $0(0 \%)$ \\
Cardiac death & 0 \\
Myocardial infarction & 0 \\
Definite stent thrombosis & 0 \\
Stroke, $\mathrm{n}(\%)$ & $0(0 \%)$ \\
Bleeding event, n (\%) & \\
Reoperation for bleeding & $4(1.9 \%)$ \\
Postoperative transfusion & $15(7.1 \%)$ \\
TIMI major/minor & $5 / 11$ \\
\hline MACE: major adverse cardiac event, TIMI: \\
thrombolysis in myocardial infarction
\end{tabular}

crease in hemoglobin concentration or $>10 \%$ decrease in hematocrit) or no observed blood loss $(>4 \mathrm{~g} / \mathrm{dL}$ decrease in hemoglobin concentration or a $>12 \%$ decrease in hematocrit)] (11).

The data are presented as the mean \pm SD. The categorical
Table 2. Characteristics of Surgical Procedure.

\begin{tabular}{|c|c|}
\hline & $\mathrm{n}=210$ \\
\hline \multicolumn{2}{|l|}{ Cardiac risk stratification, $\mathrm{n}$} \\
\hline Vascular & 21 \\
\hline Intermediate & 89 \\
\hline Low & 100 \\
\hline \multicolumn{2}{|l|}{ Type of surgical procedure } \\
\hline Vascular surgery & 21 \\
\hline Intraperitoneal surgery & 47 \\
\hline Intrathoracic surgery & 14 \\
\hline Carotid endarterectomy & 1 \\
\hline Head and neck surgery & 3 \\
\hline Orthopedic surgery & 5 \\
\hline Prostate surgery & 3 \\
\hline Endovascular aneurysm repair & 16 \\
\hline Endoscopic procedures & 51 \\
\hline Superficial procedure & 40 \\
\hline Eye surgery & 8 \\
\hline Breast surgery & 1 \\
\hline Malignancy & $95(45.2 \%)$ \\
\hline \multicolumn{2}{|l|}{ Hemorrhagic risk, $n$} \\
\hline High & 11 \\
\hline Intermediate & 88 \\
\hline Low & 111 \\
\hline \multicolumn{2}{|l|}{ Term after DES implantation } \\
\hline $0-1 \mathrm{y}, \mathrm{n}$ & 45 \\
\hline $1-2 y, n$ & 50 \\
\hline $2 y-, n$ & 115 \\
\hline \multicolumn{2}{|c|}{ Duration of discontinuation of all APT, day } \\
\hline Before surgical prucedure & $7.6 \pm 3.1$ \\
\hline After surgical procedure & $4.5 \pm 5.9$ \\
\hline
\end{tabular}

variables are expressed as numbers and percentages.

\section{Results}

The patient characteristics at the time of the surgical procedure and the medications that were administered before the interruption of APT are shown in Table 1. The mean age was $71 \pm 8$ years, and the majority $(84.3 \%)$ were men. The mean number of implanted DESs was $2.1 \pm 1.3$.

The characteristics of the surgical procedures are shown in Table 2. The mean interval from the last DES implantation to the surgical procedure was $31.9 \pm 23.0$ months (45 procedures within 1 year; 50 procedures from 1 to 2 years; and 115 procedures in a period of more than 2 years). The administration of all oral APT agents was stopped in the perioperative period, and the mean duration of the interruption was from 7.6 \pm 3.1 days before the procedure to $4.5 \pm 5.9$ days after the procedure.

Table 3 shows the clinical events that occurred within 30 days of surgery. No case developed MACE $(0 / 210 ; 0 \%)$ or suffered a stroke within the period. Four cases required reoperation for bleeding within 30 days $(4 / 210 ; 1.9 \%)$. Postoperative bleeding occurred 8 days and 10 days after surgery in 2 patients who underwent an endoscopic submucosal dissection for early-stage gastric cancer and 6 days after surgery in 1 patient who underwent an endoscopic mucosal resection for a rectal carcinoid. A postoperative hematoma was revealed by computed tomography just after surgery in 1 pa- 
tient who underwent a tumorectomy for a meningioma. The reoperations of all of the patients were successful and they all survived to discharge.

No patients required the cessation of heparin administration due to suspected serious heparin-induced thrombocytopenia.

\section{Discussion}

In this report, there were no cases of MACE after 210 non-cardiac surgical procedures with unfractionated heparin during the interruption of all APTs among the DES patients. The frequency of significant bleeding events was not thought to exceed that which is commonly accepted.

Recent reports have shown that the risk of adverse cardiac events was significantly increased after non-cardiac surgery in patients who had previously undergone DES implantation (1). The perioperative cardiac risk among the DES patients is considered to be mainly related to the interruption of APT and a surgery-induced prothrombotic state $(2-5,12)$. Therefore, the continuation of APT has been considered to be beneficial when the surgical bleeding risk is not prohibitive (12), and guidelines recommend the continuation of APT in the perioperative period when possible $(7-9,13)$. However, a cardiologist often encounters situations in which a surgeon is hesitant to continue APT and requests the temporary cessation of APT for surgery to reduce the risk of perioperative bleeding. Although the risk of perioperative bleeding that is associated with the continuation of APT has to be balanced against the perioperative cardiac risk that is associated with the interruption of APT, the effectiveness of continuing APT in reducing perioperative cardiac events in patients who have previously undergone coronary stenting remains unclear $(14,15)$. We have to admit that there is still not enough data to strongly reject the request. There is a wide variety of potential approaches, and eventually, perioperative management on an individual case-by-case basis will be required $(2,16,17)$.

In the clinical setting, heparin has been widely used in perioperative management as a bridging therapy during the interruption of APT. However, data regarding the administration of heparin in this period are scarce, and the value of perioperative heparin therapy remains to be elucidated $(2,6-8,16)$. Some prior reports concluded that heparin bridging therapy might be ineffective because some degree of platelet inhibition seemed to be required to prevent stent thrombosis (6). Furthermore, other reports have indicated an association between perioperative cardiac events and the administration of unfractionated heparin (18-20). Vicenzi et al. evaluated 103 non-cardiac surgeries within 1 year after coronary stenting and reported a high incidence of perioperative adverse cardiac events, particularly in patients who received unfractionated heparin (18). Although they also suggested caution in interpreting the positive association between the administration of unfractionated heparin and the occurrence of postoperative cardiac events, Dent et al. referred to the possibility of "heparin rebound" - a period of hypercoagulability after the abrupt cessation of unfractionated heparin before surgery (19). Anwarddin et al. assessed the cardiac risk of surgery in 606 patients after DES implantation, and noted that heparin was administered intravenously in about $20 \%$ of the patients before surgery (20). They showed that the intravenous administration of heparin in the preoperative period might increase the risk of adverse cardiac events. They also described the heparin rebound phenomenon and platelet activation caused by the administration of unfractionated heparin as possible explanations (20-22). Contrary to those results, no MACE occurred within 30 days of non-cardiac surgery in 210 DES cases received unfractionated heparin in our study. The level of perioperative cardiac risk peaks immediately after surgery $(12,23)$. The main cause of this might be a prothrombotic state associated with surgical stress. Heparin does not have an antiplatelet effect, but could control the hypercoagulable state after surgery, which might prevent postoperative cardiac events. Our local institutional practice guideline recommended that heparin infusion be resumed as soon as possible after surgery, which might have resulted in the low frequency of perioperative cardiac events in contrast to other reports $(18,20)$. Our results might not be sufficient to demonstrate the effect of unfractionated heparin, but at the very least can support that heparin, as administered within our experiments, was not harmful.

In this study, reoperation for bleeding was only required in 4 cases $(1.9 \%)$ and postoperative transfusion was performed in 15 cases $(7.9 \%)$. As in a previous heparin study (18), the frequency of significant bleeding was not thought to exceed that which is commonly accepted (6).

There are several limitations associated with the present study. First, this was a single center study of a relatively small population. Second, we could not compare the study group to patients who did not receive heparin or in whom the administration of APT was continued. Therefore we cannot deny the existence of other factors that related to the study results. Third, the interval from last DES implantation to the surgical procedure was relatively long in the majority of cases, which might be associated with a low incidence of MACE. Fourth, the decisions to consult cardiologists and require the interruption of APT for the procedure were basically left to the discretion of the attending surgical team. Therefore it might not be appropriate to perform some procedures with the interruption of APT. Fifth, we could not deny the existence of silent stent occlusion. Notwithstanding these limitations, to date, only a few investigations have assessed the perioperative administration of heparin and this clinical study will be meaningful in the accumulation of data regarding this issue. It is not recommended to administer heparin during the interruption of all ATPs for noncardiac surgery; however, there are insufficient data to determine the value. Furthermore, there is wide variability in the circumstances surrounding the administration of APT among different countries. Large multicenter prospective clinical in- 
vestigations and further discussion about this issue are required.

In conclusion, our data showed the potential for perioperative management with unfractionated heparin administration in Japanese patients who had previously undergone DES implantation who required non-cardiac surgery with the interruption of all APTs.

The authors state that they have no Conflict of Interest (COI).

\section{References}

1. Berger PB, Kleiman NS, Pencina MJ, et al. Frequency of major noncardiac surgery and subsequent adverse events in the year after drug-eluting stent placement results from the EVENT (Evaluation of Drug-Eluting Stents and Ischemic Events) Registry. JACC Cardiovasc Interv 3: 920-927, 2010.

2. Abualsaud AO, Eisenberg MJ. Perioperative management of patients with drug-eluting stents. JACC Cardiovasc Interv 3: 131$142,2010$.

3. Grines CL, Bonow RO, Casey DE, et al. Prevention of premature discontinuation of dual antiplatelet therapy in patients with coronary artery stents: a science advisory from the American Heart Association, American College of Cardiology, Society for Cardiovascular Angiography and Interventions, American College of Surgeons, and American Dental Association, with representation from the American College of Physicians. Circulation 115: 813-818, 2007.

4. Riddell JW, Chiche L, Plaud B, Hamon M. Coronary stents and noncardiac surgery. Circulation 116: e378-e382, 2007.

5. Tanaka A, Sakakibara M, Ishii H, et al. The risk of adverse cardiac events following minor surgery under discontinuation of all antiplatelet therapy in patients with prior drug-eluting stent implantation. Int J Cardiol 172: e125-e126, 2014.

6. Singla S, Sachdeva R, Uretsky BF. The risk of adverse cardiac and bleeding events following noncardiac surgery relative to antiplatelet therapy in patients with prior percutaneous coronary intervention. J Am Coll Cardiol 60: 2005-2016, 2012.

7. Douketis JD, Spyropoulos AC, Spencer FA, et al. Perioperative management of antithrombotic therapy: Antithrombotic Therapy and Prevention of Thrombosis, 9th ed: American College of Chest Physicians Evidence-Based Clinical Practice Guidelines. Chest 141: e326S-e350S, 2012.

8. Fleisher LA, Beckman JA, Brown KA, et al. 2009 ACCF/AHA focused update on perioperative beta blockade incorporated into the ACC/AHA 2007 guidelines on perioperative cardiovascular evaluation and care for noncardiac surgery. J Am Coll Cardiol 54: e13e118, 2009.

9. Windecker S, Kolh P, Alfonso F, et al. 2014 ESC/EACTS Guidelines on myocardial revascularization: The Task Force on Myocardial Revascularization of the European Society of Cardiology (ESC) and the European Association for Cardio-Thoracic Surgery (EACTS) Developed with the special contribution of the European
Association of Percutaneous Cardiovascular Interventions (EAPCI). Eur Heart J 35: 2541-2619, 2014.

10. Chassot PG, Delabays A, Spahn DR. Perioperative antiplatelet therapy: the case for continuing therapy in patients at risk of myocardial infarction. Br J Anaesth 99: 316-328, 2007.

11. Bovill EG, Terrin ML, Stump DC, et al. Hemorrhagic events during therapy with recombinant tissue-type plasminogen activator, heparin, and aspirin for acute myocardial infarction. Results of the Thrombolysis in Myocardial Infarction (TIMI), Phase II Trial. Ann Intern Med 115: 256-265, 1991.

12. Brilakis ES, Patel VG, Banerjee S. Medical management after coronary stent implantation: a review. JAMA 310: 189-198, 2013.

13. Poldermans D, Bax JJ, Boersma E, et al. Guidelines for preoperative cardiac risk assessment and perioperative cardiac management in non-cardiac surgery. Eur Heart J 30: 2769-2812, 2009.

14. Hawn MT, Graham LA, Richman JS, et al. Risk of major adverse cardiac events following noncardiac surgery in patients with coronary stents. JAMA 310: 1462-1472, 2013.

15. Tokushige $A$, Shiomi $H$, Morimoto $T$, et al. Incidence and outcome of surgical procedures after coronary bare-metal and drugeluting stent implantation: a report from the CREDO-Kyoto PCI/ CABG registry cohort-2. Circ Cardiovasc Interv 5: 237-246, 2012.

16. Korte W, Cattaneo M, Chassot PG, et al. Peri-operative management of antiplatelet therapy in patients with coronary artery disease: joint position paper by members of the working group on Perioperative Haemostasis of the Society on Thrombosis and Haemostasis Research (GTH), the working group on Perioperative Coagulation of the Austrian Society for Anesthesiology, Resuscitation and Intensive Care (OGARI) and the Working Group Thrombosis of the European Society for Cardiology (ESC). Thromb Haemost 105: 743-749, 2011.

17. Alshawabkeh LI, Banerjee S, Brilakis ES. Systematic review of the frequency and outcomes of non-cardiac surgery after drugeluting stent implantation. Hellenic J Cardiol 52: 141-148, 2011.

18. Vicenzi MN, Meislitzer T, Heitzinger B, Halaj M, Fleisher LA, Metzler H. Coronary artery stenting and non-cardiac surgery: a prospective outcome study. Br J Anaesth 96: 686-693, 2006.

19. Dent H, Lekic Z, Vicenzi M. Unfractionated heparin and coronary artery stenting. Br J Anaesth 97: 582, 2006.

20. Anwaruddin S, Askari AT, Saudye H, et al. Characterization of post-operative risk associated with prior drug-eluting stent use. JACC Cardiovasc Interv 2: 542-549, 2009.

21. Theroux P, Waters D, Lam J, Juneau M, McCans J. Reactivation of unstable angina after the discontinuation of heparin. N Engl $\mathrm{J}$ Med 327: 141-145, 1992.

22. Xiao Z, Theroux P. Platelet activation with unfractionated heparin at therapeutic concentrations and comparisons with a lowmolecular-weight heparin and with a direct thrombin inhibitor. Circulation 97: 251-256, 1998.

23. Alshawabkeh LI, Prasad A, Lenkovsky F, et al. Outcomes of a preoperative "bridging" strategy with glycoprotein IIb/IIIa inhibitors to prevent perioperative stent thrombosis in patients with drug-eluting stents who undergo surgery necessitating interruption of thienopyridine administration. EuroIntervention 9: 204-211, 2013.

(C) 2016 The Japanese Society of Internal Medicine

http://www.naika.or.jp/imonline/index.html 